\title{
Rapid Evaluation Method for Accuracy of Range Estimation of Pure Electric Vehicle Range Estimation Based on CLTC-P
}

\author{
Tianlu Dai ${ }^{1}$, Boya Zhou ${ }^{1}$, Yanyi Zhang ${ }^{1}$, Guang Chen $^{1}$, Peng Liu ${ }^{1}$ \\ ${ }^{1}$ China Automotive Research\&Center Co,300300, Tianjin China
}

\begin{abstract}
Based on theoretical and experimental research, this paper constructs a rapid test method for estimating the accuracy of the range of pure electric vehicles based on CLTC-P, which is based on different environmental temperatures, air conditioning use, test scenarios, and high-speed operating conditions. The article further compares and analyzes a variety of mathematical statistical methods and selects certain coefficients to evaluate the accuracy of cruising range estimation, and proposes a general rapid test method and evaluation theory of cruising range estimation accuracy, which lays the foundation for establishing a unified assessment standard in industry, and promotes the development of the electric vehicle industry.
\end{abstract}

\section{Introduction}

According to consumer research, the accuracy of the remaining display is one of the indicators that consumers are more concerned about but have lower satisfaction, and there is a large room for improvement. The survey results show that in the satisfaction scores of battery performance indicators and battery life indicators, the remaining mileage display accuracy scores are not satisfactory for two consecutive years (2018 and 2019) [1-2]. Especially when the electric vehicle is in a state of high energy consumption, such as when driving at a high speed, the displayed mileage drops much faster than the actual mileage. Even when some vehicles have a relatively low State of Charge (SOC), it appears that there is still a certain amount of mileage that can be driven, but the vehicle cannot drive because of insufficient power. The above problems have increased consumers' mileage anxiety to a certain extent.

At present, the algorithm logic and display strategy of various car companies to display the cruising range are different, and even the display strategies of different models in the same car company are completely different. At present, the research on whether the vehicle's cruising range is accurate at home and abroad generally focuses on the analysis of the error between the remaining mileage and the actual remaining range. However, there is no unified conclusion on whether the error can accurately evaluate the accuracy of the remaining cruising range[34].

Similar to fuel-powered vehicles, there is a big difference between the display of cruising range and the actual driving conditions of consumers. There are many reasons for the above phenomenon, the most important of which is that the current battery life of electric vehicles and official publicity are based on the test results under
NEDC conditions. This test method differs from the actual road in China from the beginning of the test. At present, there is no unified and rapid evaluation method for the estimation of vehicle cruising range in the industry, which is more in line with China's actual road conditions. Therefore, There is certain practical significance to research and develop a rapid test and evaluation method for the estimation accuracy of the range of pure electric passenger vehicles based on CLTC-P, and promote the implementation of "CLTC-P" in the field of pure electric passenger vehicles. At the same time, it is committed to alleviating consumers' anxiety about the mileage of electric vehicles[5-7].

\section{Research process}

According to the needs of electric vehicle usage scenarios and regions, it is necessary to construct typical test conditions for test range estimation accuracy from multiple dimensions such as working conditions, temperature, air conditioning and test conditions.

\subsection{Selection of basic working conditions for testing}

The basic driving conditions of the automobile are an important and common basic technology of the automobile industry, and the main benchmark for the calibration and optimization of various automobile performance indicators. The existing NEDC operating conditions underestimate the energy-saving effect of new energy vehicles. The main characteristics of WLTC, such as idle time and average speed, are even more different from the actual operating conditions in my country, as shown in Figure 1. 


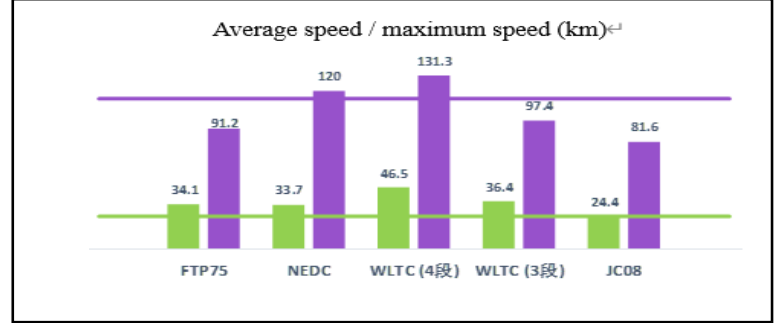

Fig1. Comparison of average speeds in different operating conditions

By comparing the driving characteristics of new energy vehicles with traditional vehicles, it can be found that new energy vehicles share the road resources with traditional vehicles, the running characteristics are highly coincident, and there is a strong similarity in speed, acceleration, and sports history. At the same time, with the gradual improvement of the charging infrastructure of the inter-city road network at this stage, consumers can drive electric vehicles to supply electricity for long distances. Moreover, after joining the plan for driving on the highway, the high-speed ratio of new energy vehicles is very close to that of traditional vehicles. Therefore, new energy vehicles have the basis for using "CLTC-P".

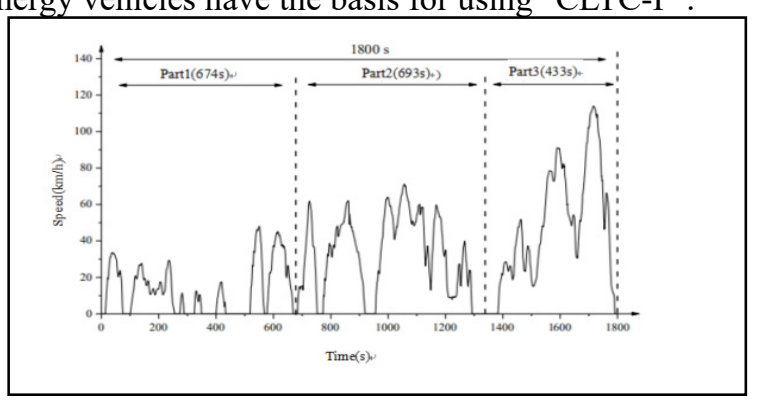

Fig2. Speed curve of CLTC-P

\subsection{The construction of rapid evaluation working condition Based on CLTC-P}

First of all, the current driving range test for electric vehicles is mainly to measure the distance traveled by the vehicle during the entire process when the battery of the electric vehicle is fully charged and the battery power is consumed until it is close to the unusable state through continuous designated test cycles. Although this method can measure and evaluate the driving range of electric vehicles to the greatest extent, the test efficiency is low and the cost is high. With the improvement and development of various technologies of electric vehicles, the emergence of vehicles with longer driving range has become possible. If the current test method is used, it will be detrimental to the development of the test work. A test method that can quickly evaluate the driving range of electric vehicles should be developed to cope with the development trend of long driving range of electric vehicles.

Secondly, in the long-term evaluation, it is found that there is a systematic error in part of the evaluation process of the cruising range evaluation test. In order to ensure the representativeness and objectivity of the evaluation results, and to ensure the high stability of the test results, it is necessary to carry out the secondary development of the key evaluation technology for the range of electric vehicles to achieve high efficiency, high accuracy, and high coverage upgrades.

Third, as mentioned above, with the popularization of electric vehicles, the charging infrastructure of the highway service area is gradually improved, and highway charging will no longer be a bottleneck problem for electric vehicles at high speeds. Therefore, it will become more and more common for consumers to drive electric vehicles through long distances on highways. Therefore, a large proportion of high-speed and constant-speed driving scenarios can be added to the test conditions, thereby forming a composite test condition.

Therefore, based on the CLTC-P test conditions, this paper conducts a research on the test method of electric vehicle driving range, and establishes a rapid test evaluation method that reflects the accuracy of electric vehicle driving range estimation under the actual operating conditions in China. It can shorten the test time and reduce the test workload under the condition of ensuring reliable test results, and improve the evaluation efficiency of the estimation accuracy of the mileage of electric vehicles. The speed curve of the composite test mode is shown in Figure 3.

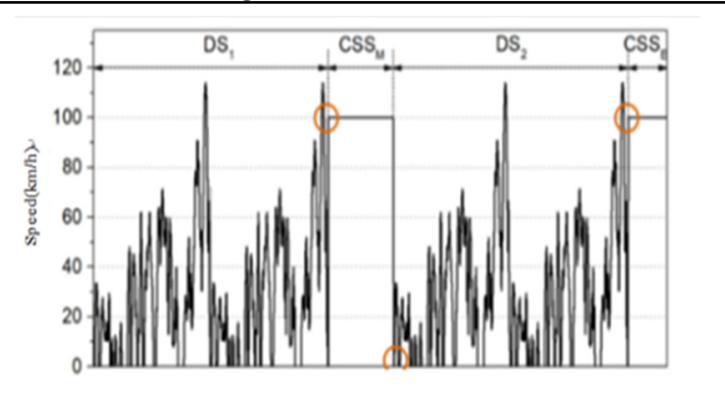

Fig3. Composite test conditions based on CLTC-P

The shortening method speed segment is composed of 2 test cycle sections and 2 constant speed sections. Among them, DS1 and DS2 are test cycle sections, which are composed of CLTC-P; CSSM and CSSE are constant speed sections, which are composed of higher constant vehicle speeds. The estimation accuracy of the cruising range of the vehicle is calculated through the test results of the two test cycles.

\subsection{Determination of typical environmental conditions}

First of all, the determination of typical environmental conditions such as laboratory environment and air conditioning settings fully takes into account china's actual climate, vehicle conditions and driving habits of Chinese drivers. Secondly, the use characteristics of air conditioners include the overall characteristics of air conditioner shutdown, cooling and heating, and the characteristics of cooling and heating use vary with temperature. The critical ambient temperature for turning on air conditioning and cooling reflects the current general habits of domestic drivers. The annual use time of car air conditioners in various regions can be approximated by combining the annual average temperature curve of each region. 


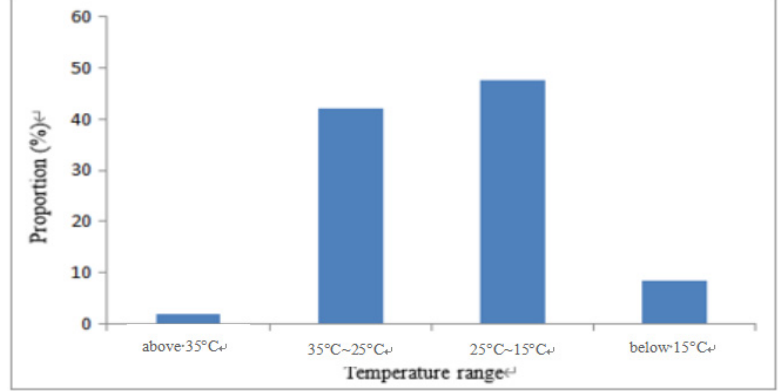

Fig4. Survey of critical ambient temperature for air conditioning and refrigeration

Through investigation and research on different cities and consumers in China, it is determined that the endurance test is divided into normal temperature endurance $\left(25^{\circ} \mathrm{C}\right)$, high temperature endurance $\left(35^{\circ} \mathrm{C}\right)$ and low temperature endurance $\left(-7^{\circ} \mathrm{C}\right)$. When conducting high-temperature or low-temperature endurance tests, the air conditioner needs to be turned on. During the hightemperature test, the interior temperature must be maintained between $23^{\circ} \mathrm{C}$ and $25^{\circ} \mathrm{C}$, and during the lowtemperature test, the interior temperature must be maintained between $20^{\circ} \mathrm{C}$ and $22^{\circ} \mathrm{C}[8]$.

\subsection{Test preparation and data collection}

The vehicle cruising range test is based on the principle of ensuring the authenticity of the data and the sequence of the test. The test first conducts the taxi resistance test, then conducts the cruising range bench test, and performs data collection simultaneously.

The taxiing resistance test should ensure that the test environment meets the requirements of the specification. All vehicles are sliding in neutral, and multiple tests can be used to obtain the sliding resistance parameters, provide a resistance curve for the energy consumption test, and confirm the sliding resistance curve on the drum.

The cruising range test loads the vehicle to a specified load, immerses the car to a specified temperature, and uses the composite test conditions constructed in this paper to test the cruising range of the entire vehicle.

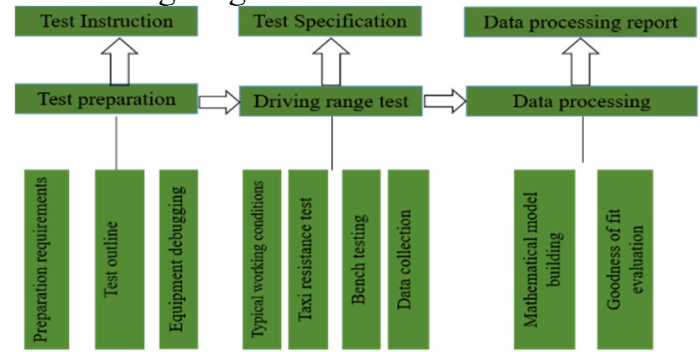

Fig5. The actual vehicle test and data collection process

The actual driving mileage and meter display cruising range data collection work simultaneously: during the test, record the remaining mileage of the meter display during the driving process of the vehicle, and record it as $y_{i}$. The actual mileage of the vehicle is calculated by subtracting the vehicle's driving mileage and time integral from the vehicle's final mileage to calculate the actual remaining mileage of the vehicle, which is recorded as $\hat{y}_{i}$.

The specific test preparation, development and data acquisition process are shown in Figure 5 above.

According to the comparison of the actual mileage and the collected cruising mileage data of the meter display, the relationship between the actual remaining mileage of the electric vehicle and the remaining mileage of the meter display is analyzed.

\subsection{Selection of data analysis methods}

In this paper, the corresponding mathematical models of the two are established, and the accuracy of the cruising range is obtained according to the best fitting evaluation. The goodness of fit in statistics describes the quantitative relationship between two variables. The closer the observation points are to the regression line, the better the fit, and the worse the converse. Commonly used methods to describe the degree of fitting include distance evaluation method and correlation coefficient method.

Commonly used distances include European distance, Manhattan distance, Chebyshev distance, etc. Through data analysis, we know that the Manhattan distance and Chebyshev distance are easily affected by extreme points, the error is large, and the mathematical model is not consistent with the actual situation, so it can not reflect the accuracy of battery life estimation. In any case, the determination coefficient can more accurately reflect the degree of deviation between a series of actual values and theoretical values. The comparison is shown in Figure 6 .

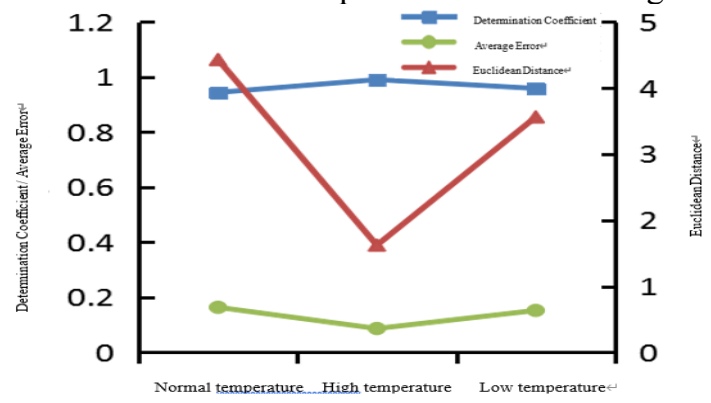

Fig6. Accuracy characterization by different statistical methods

The determination coefficient is a digital characteristic that can represent the relationship between a random variable and multiple random variables, and a statistical indicator that shows the reliability of the change of the dependent variable. The calculation formula is:

$$
R^{2}=1-\frac{\sum\left(y_{i}-\hat{y}_{i}\right)^{2}}{\sum\left(y_{i}-\bar{y}\right)^{2}} \text {. }
$$

The closer is to 1 , it indicates that the better the fitting degree, the higher the accuracy of the cruising range estimation; on the contrary, the closer $R^{2}$ is to 0 , the worse the fitting degree is, and the worse the accuracy of the cruising range estimation. When $R^{2} \leq 0$, it is considered that the degree of fitting is extremely poor, indicating that the displayed cruising range has relatively lost reference significance.

This paper uses the determination coefficient $R^{2}$ to evaluate the goodness of fit[9].

In summary, combined with the investigation of consumer usage scenarios, the strategic research on the geographical coverage of product launches by automotive companies, and the comprehensive analysis of consumer requirements for driving comfort, the final battery life based on CLCT-P is finally determined. The combination 
of scenario scenarios for rapid evaluation of mileage estimation accuracy is shown in Table 1 below:

Table1. Typical working condition combinations for evaluation

\begin{tabular}{|c|c|c|c|c|}
\hline $\begin{array}{c}\text { Operating } \\
\text { conditions }\end{array}$ & Temperature & $\begin{array}{c}\text { Air } \\
\text { conditioning }\end{array}$ & Scene & Statistics \\
\hline \multirow{3}{*}{$\begin{array}{c}\text { Composite } \\
\text { test } \\
\text { conditions } \\
\text { based on } \\
\text { CLTC-P }\end{array}$} & $\begin{array}{c}\text { Normal } \\
\text { temperature } \\
\left(25^{\circ} \mathrm{C}\right)\end{array}$ & Off & $\begin{array}{l}\text { Immerse } \\
\text { vehicles }\end{array}$ & \multirow{3}{*}{$\begin{array}{l}\text { Determinati } \\
\text { on } \\
\text { Coefficient }\end{array}$} \\
\hline & $\begin{array}{c}\text { High } \\
\text { temperature } \\
\left(35^{\circ} \mathrm{C}\right)\end{array}$ & $\begin{array}{c}\text { On } \\
23-25^{\circ} \mathrm{C}\end{array}$ & $\begin{array}{l}\text { Immerse } \\
\text { vehicles } \\
\text { Lighting }\end{array}$ & \\
\hline & $\begin{array}{c}\text { Low } \\
\text { temperature } \\
\left(-7^{\circ} \mathrm{C}\right)\end{array}$ & $\begin{array}{c}\text { On } \\
20-22^{\circ} \mathrm{C}\end{array}$ & $\begin{array}{l}\text { Immerse } \\
\text { vehicles }\end{array}$ & \\
\hline
\end{tabular}

\section{Test results of the accuracy of range estimation}

\subsection{Test results of some vehicle models based on CLTC-P}

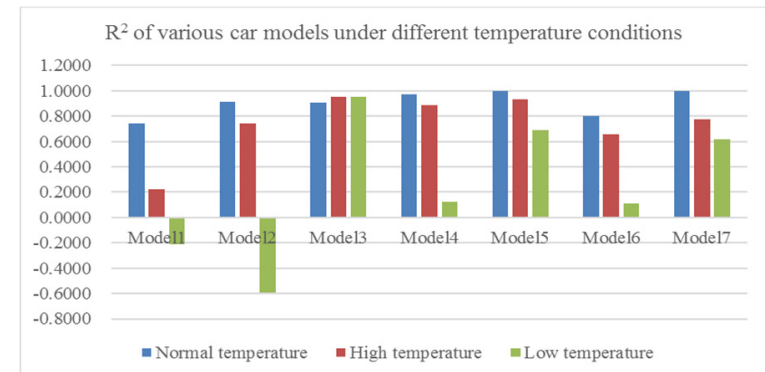

Fig7. Test results of some vehicle models under full CLTC-P Figure 7 shows the test results of the estimation accuracy of the cruising range of seven models under different temperature conditions. It can be seen from the figure:

In the normal temperature environment, the determination coefficient $R^{2}$ of all vehicle models is greater than 0.6 , and the average value reaches 0.90 , indicating that the vehicle's apparent cruising range and the actual remaining range fit well at normal temperature. The estimated accuracy of the cruising range is also high, and the overall performance is very good.

In the high-temperature environment, the average value of the determination coefficient $R^{2}$ of all models is 0.74 . Only the determination coefficient $R^{2}$ of the 03 and 05 models is greater than 0.90 , while the estimation accuracy of the cruising range of the 01 model is the worst, only 0.22 . The overall performance of the mileage estimation accuracy has declined compared to normal temperature, and the gap appears.

In a low-temperature environment, the average value of the determination coefficient $R^{2}$ for all models is 0.24 , and the overall performance is poor. The determination coefficient $R^{2}$ of the 01 model and the 02 model is less than 0 , indicating that the fitted range of the displayed range of the vehicle and the actual remaining range is extremely poor, and the displayed range of the range has relatively lost its reference significance. The average value of the determination coefficient of the other five models is 0.50 . Only the determination coefficient of the 03 model is 0.95 , which is higher than 0.8 . It can be seen that the accuracy differentiation between different models under low temperature environment More serious. When testing in high and low temperature environments, it is required to turn on the air conditioner synchronously, which will consume a large amount of battery power[10], which will bring greater challenges to the accuracy of cruising range estimation.

Looking at the overall situation under three temperature environments, the best overall performance is the 03 model. Its apparent cruising range under normal temperature, high temperature and low temperature environment is not much different from the actual cruising range, and the determination coefficient $R^{2}$ is greater than 0.90 . The best performance under high temperature environment (the highest), followed by the low temperature, the under normal temperature environment with relatively poor performance also reached 0.91 , indicating that the cruising range shown by it is higher in different temperature environments Reference value. It can be seen from the figure that only the three models 03 , 05 and 07 have a determination coefficient $R^{2}$ greater than 0.60 in the three temperature environments, and the determination coefficient $R^{2}$ of the other models differs greatly in different ambient temperatures. This type of vehicle has a large change in the accuracy of the estimated remaining mileage in different seasons, so it is easy to bring mileage anxiety to consumers.

\subsection{Evaluation test results of $R^{2}$ based on rapid evaluation of cruising range estimation accuracy}

For the accuracy of cruising range estimation, the composite working condition (as shown in Figure 3) is obviously equivalent to adding jump evaluation points, while adding a longer high-speed working condition, which brings higher accuracy to cruising range estimation Challenge. In this paper, three representative models are selected to compare the evaluation accuracy of the endurance estimation of the full operating mode and the composite operating mode. The specific results are shown in Figure 8 below.

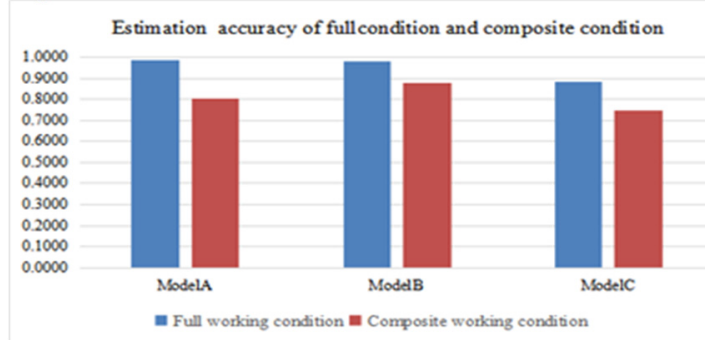

Fig8. Comparison of the accuracy of endurance estimation between the full operating mode and the composite operating mode

Figure 8 shows the comparison of the test results of the estimated accuracy $R^{2}$ of the cruising range of the three models under the CLTC-P and the composite conditions method based on the constant speed section of $100 \mathrm{~km} / \mathrm{h}$. As can be seen from the comparison in the figure, the accuracy of the estimated range $R^{2}$ of the three models in single full CLTC-P is shown in the table below. However, under the composite conditions, because of the existence of three trip, the estimated accuracy $R^{2}$ of cruising range has been greatly reduced. The three models 
with the largest decrease in accuracy $R^{2}$ are model A ($18.51 \%)$, and the smallest is model B $(-10.08 \%)$, the average decrease is $14.61 \%$. It shows that the composite working conditions bring higher requirements to the accuracy $R^{2}$.

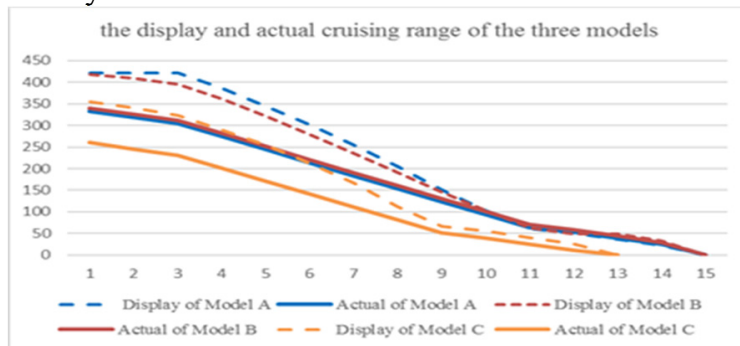

Fig9. The mileage of three models under normal operating conditions at normal temperature

Figure 9 shows the cruising range and actual cruising range of the three models under normal temperature environment, based on the composite operating conditions of the constant speed section of $100 \mathrm{~km} / \mathrm{h}$. The curves fitted by the three sets of data all show the shape of the bell mouth, indicating that the accuracy of the estimated battery life in the car is low. In the later stage of the test, the fitting curves of the three vehicles gradually tended to converge, that is, the fit between the displayed endurance curve of each car and the actual endurance curve was getting better and better, indicating that the three car meter endurance mileages are getting closer and closer to the actual endurance mileage, and the accuracy of the endurance mileage estimation is improved. This is because these three models have a self-learning mode. This is because these three models have a self-learning mode in the cruising range estimation strategy[11]. This mode can correct the current remaining mileage according to the previous mileage energy consumption, thereby improving the accuracy of displaying the remaining mileage estimation[12]. This model is currently one of the most important and widely used models for electric passenger vehicle mileage estimation.

\section{Conclusion}

Based on the previous research situation, this paper builds a rapid test evaluation method based on CLTC-P, which aims at the accuracy of the cruising range estimation formed by the combination of different environmental temperatures, air conditioning use, test scenarios and mathematical statistical methods. Some research results have been achieved

4.1.1 The estimated accuracy of the cruising range of electric vehicles at different ambient temperatures is also different, with normal temperature being the best, high temperature being the second, and low temperature being the worst. Under the normal temperature environment, the determination coefficient $R^{2}$ of each vehicle model is generally greater than 0.6 , and the average value reaches 0.90. In high-temperature environments, the difference in the accuracy of the estimates of various models appears. In a low-temperature environment, the overall performance is poor and the differentiation is more serious. The average value of the determination coefficient $R^{2}$ of all vehicle models is 0.24 , and even the determination coefficient $R^{2}$ of some vehicle models is less than 0 , indicating that the cruising range shown has relatively lost its reference significance..

4.1.2 Because of the existence of three trip points and longer high-speed operating conditions, the compound operating conditions impose higher requirements on the accuracy of cruising range estimation. The average decrease in the estimated accuracy $R^{2}$ of the test vehicle is $14.61 \%$. Tests show that the three vehicles have considered the self-learning mode in the calculation method of the meter display cruising range, which makes the meter display cruising range more and more close to the actual cruising range, which has a positive impact on improving the accuracy of the cruising range estimation.

This article can further study the establishment of corresponding scoring rules for the estimation accuracy of cruising range, and lay the foundation for establishing a unified evaluation standard in the industry.

\section{Acknowledgment}

Project: Research and application of the technology for rapid evaluation of pure electric vehicle cruising range (10219-09).

\section{References}

1. "2018 China New Energy Vehicle Industry Consumer Survey Report."

2. "2019 China New Energy Vehicle Industry Consumer Survey Report."

3. J.L Yibing, L Binbin, H Ning, G zhichao, M Yuchen. "Estimation for SOC of batteries for EVs and range showing and alerting". vol54(02) Chinese Labat Man, 2017, pp. 65-69+93.

4. D.C Qiujin. "Research on SOC Estimation and Range of Electric Vehicle Based on Simulink". Chang'an University, 2018.

5. J Jiuchun, R Haijun, S Bingxiang, W Leyi, G Wenzhong, Z Weige, "A low-temperature internal heating strategy without lifetime reduction for largesize automotive lithium-ion battery pack" Applied Energy, 2018. pp 257-266,.

6. W Jian, L Tong, Z Hao, L Yanxiang, Z Guangquan, "Research on Modeling and SOC Estimation of Lithium Iron Phosphate Battery at Low Temperature,Energy " Procedia,vol 152, pp556561,2018 .

7. M Jinhao, R Mattia, Anirudh, A Budna, L Guangzhao, Maciej Swierczynski, S Daniel-Ioan, Remus "Teodorescu,Low-complexity online estimation for LiFePO4 battery state of charge in electric vehicles" Journal of Power Sources, vol 395,2018, pp280-288.

8. "EV-TEST Electric Vehicle Evaluation Rules (2019)"

9. J JunPing . Statistics, Beijing: Tsinghua University Press,2004,298-299.

10. X Guodong,C Lei,B Guanglong,"A review on battery 
thermal management in electric vehicle application " Journal of Power Sources,vol 367,2017,pp 90-105.

11. C Fleischer, W Waag, Z Bai, D Uwe Sauer,"On-line self-learning time forward voltage prognosis for lithium-ion batteries using adaptive neuro-fuzzy inference system " ,Journal of Power Sources, vol243,2013,pp 728-749.

12. C Sbarufatti, M Corbetta, M Giglio, F Cadini, "Adaptive prognosis of lithium-ion batteries based on the combination of particle filters and radial basis function neural networks",Journal of Power Sources, vol 344,2017,pp 128-140. 